

\title{
Immunosuppression-lipid metabolism interplay and medicinal plants in atherosclerosis: a review
}

\author{
Fahimeh Moradi ${ }^{1}$, Robert D. E. Sewell ${ }^{2}$, Zahra Lorigooini ${ }^{2}$, Mahmoud Rafieian-Kopaei ${ }^{2}$ \\ ${ }^{1}$ Medical Plants Research Center, Basic Health Sciences Institute, Shahrekord University of Medical \\ Sciences, Shahrekord, Iran \\ ${ }^{2}$ Cardiff School of Pharmacy and Pharmaceutical Sciences, Cardiff University, Cardiff, CF10 3NB. \\ Wales, U.K.
}

Corresponding author: $\underline{\text { rafieian@yahoo.com }}$

Tel: +989131811842

\begin{abstract}
:
Atherosclerosis is a chronic arterial disease responsible for the majority of vascular related deaths throughout the world. Immune cells and inflammation in conjunction with hyperlipidemia, play a key role in atherosclerosis development. Regarding the low efficacy of synthetic drugs and also the associated negative side effects which can adversely influence health-related quality of life, looking for natural, affordable and non-toxic substances appears to be necessary. Plant-derived natural products play a critical role in prevention and treatment of atherosclerosis. In this review, we aimed to outline the most important medicinal herbs effective on atherosclerosis through an impact on the immune system.
\end{abstract}

Keywords: Atherosclerosis, Cardiovascular disease, Medicinal plants, Immune system, 


\section{Introduction:}

According to WHO estimates, cardiovascular disease (CVD)-related events such as myocardial infarction (MI) and stroke are responsible for one-third of deaths worldwide (1, 2). Due to the increase in obesity and diabetes, CVD is expected to rise to roughly $44 \%$ by 2030 so inflicting a great deal of pressure on healthcare services $(3,4)$.

Atherosclerosis, as the leading cause of CVD, is a chronic inflammatory disorder of the vasculature (5) that is affected by genetic susceptibility and also different lifestyle factors including age, gender, stress, smoking and dietary habits $(6,7)$. However, in spite of lifestyle modifications and the development of new pharmacological approaches for reducing plasma concentrations of total cholesterol, CVD remains a predominant cause of mortality throughout the world $(8,9)$.

Not only as a lipid accumulation disorder, but also as a chronic inflammatory process of arterial walls, atherosclerosis is induced by factors including an interaction of lipid metabolism imbalance, abnormal immune response and genetic alterations (10-12). In this regard, components of both innate and adoptive immune systems participate in atherosclerosis pathology $(12,13)$. Briefly, LDL-cholesterol deposits and accumulates in the arterial wall and then LDL is oxidized (OxLDL). It has been proposed that the formation of OxLDL is a critical incident that exacerbates inflammation in the arterial wall. Due to overexpression of adhesion molecules, different types of leukocyte and also monocytes migrate into the vascular wall and produce chemokines and pro-inflammatory cytokines. The uptake of OxLDL is performed by monocyte-derived macrophages through scavenger receptors transforming the cells into lipid-laden foam cells $(13,14)$. The differentiation into lipid-laden foam cells is crucial in the development of atherosclerosis (15). 
The first visible lesion in the development of atherosclerosis is a fatty streak which contains lipids and various immune cells consisting of $\mathrm{T}$ lymphocytes, dendritic cells, and macrophages. Progressed atherosclerotic lesions involve migrated smooth muscle cells (SMCs), debris, apoptotic cells, and extracellular matrix comprising collagen and proteoglycans (13). It is thought that B lymphocytes and their secreted immunoglobulins, including $\operatorname{IgG}$ and $\operatorname{IgM}$, are associated with atherogenesis. Unstable atherosclerotic plaques may suddenly break off and give rise to a life-threatening coronary thrombosis. Substantial features of indolent plaque include infiltration of many inflammatory cells, a large lipid core, and a thin fibrous cap $(16,17)$.

Pro-inflammatory cytokines like interleukin 1 and tumor necrosis factor regulate the expression of adhesion molecules involved in early and late stages of atherogenesis. IL-1 and TNF can stimulate the production of growth factors, including fibroblast growth factors (FGF) and platelet derived growth factor (PDGF). These growth factors attract smoothmuscle cells into the tunica intima resulting in inflammation. However, other cytokines and growth factors may be important in developing a more advanced form of atherosclerosis with a decreased susceptibility of plaque to rupture. For example, interstitial collagen production is stimulated by transforming growth factor-b (TGF-b) while it is neutralized by interferon-g (IFN-g) $(18,19)$.

Altogether, prevention of atherosclerosis might be achieved by intervening at each step or the inflammatory-associated factors involved in the atherogenesis process (14).

Nevertheless, regarding the low efficacy of synthetic drugs and also their associated adverse effects including dry mouth, emotional distress, vertigo and stomach disturbances which can adversely affect health-related quality of life, looking for natural, affordable and non-toxic substances appears to be necessary. Currently, plants are widely used in traditional medicine 
to treat a broad spectrum of illnesses and approximately $80 \%$ of the emerging world population depends upon traditional medicine for primary health care (20). In this review, we aimed to outline the most important medicinal herbs effective on atherosclerosis through an impact on the immune system.

\section{Medicinal Plants and Atherogenesis}

Targeted intervention strategies, including anti-inflammatory and immunomodulatory approaches or lipid metabolism modifier-based procedures have been employed to control atherosclerosis $(21,22)$. Traditional medicine, which is profoundly ingrained in history, has been widely used to prevent and treat a variety of diseases including atherosclerosis. Medicinal herbs are supposed to suppress immune responses and any consequent inflammation. Having knowledge of medicinal plants employed in atherosclerosis management is very helpful to determine the possible use of these herbs in cytokine or lipid metabolism-based therapies. In the following discourse, the potential clinical predictive value of some medicinal herbs in reducing proinflammatory cytokine expression or modifying lipid metabolism in the management of the progression and complications of atherosclerosis will be explained.

\section{Turmeric}

Turmeric (Curcuma longa) or "Indian saffron" is a spice that has also been used in some medicinal preparations and as a cosmetic for many years in Southeast Asian countries (23). One of the most well-known plant-based natural compounds, called Curcumin, is extracted from the rhizome of the Turmeric plant. Curcumin is a hydrophobic, low molecular weight polyphenol and also a major active component in turmeric. It has been shown to be effective 
in the treatment of age-associated diseases like atherosclerosis by exerting antiinflammatory, anti-oxidative, and anti-atherosclerotic effects (24). Studies have indicated that curcumin significantly represses $\mathrm{MCP}-1$ production and increases cholesterol efflux through inhibition of JNK pathways and it has been concluded that curcumin protects the vascular system against developing atherosclerotic lesions (25).

\section{Cassia occidentalis}

Different parts of the annual herb Cassia occidentalis Linn. (Caesalpiniaceae) have a variety of medicinal impacts including anti-inflammatory, antihepatotoxic (26), antibacterial (27), antiplasmodial (28) and antidiabetic (29) properties. A leaf aqueous extract of this herb has been used for the treatment of cardiovascular diseases in African traditional medicine. According to studies, treating animal models with a leaf aqueous extract of Cassia occidentalis significantly increased the faecal excretion of cholesterol by transformation of cholesterol into biliary acids in rats subjected to a feeding regime high in cholesterol. Furthermore, by increasing LCAT activity, aqueous extract of Cassia occidentalis enriched the HDL-C out of cholesterol esters. Taken together, studies have provided evidence of involving the aqueous extract of $C$. occidentalis in lipid metabolism, even though safety has yet to be explored (28).

\section{Cornelian cherry}

Cornus mas L. (Cornelian cherry) is a flowering plant in the dogwood family. Due to its high antioxidant and anti-inflammatory properties, Cornelian cherry is considered a medicinal herb and applied in therapeutic approaches against a variety of conditions including inflammatory bowel disease, fever, kidney stones, urinary tract infections as well as bleeding. Moreover, several studies have demonstrated that Cornus mas L. has a protective role against atherosclerosis and give rises to a reduction of fat accumulation in the liver (30). 


\section{Humulus japonicas}

Humulus japonicas $(\mathrm{HJ})$ is a decorative plant in the Cannabaceae family and is a native plant in temperate parts of Asia and the tropical area of Vietnam. According to a study conducted in 2016 by Lim et al, Humulus japonicas significantly prevented atherogenesis by repressing inflammation and the development of atherosclerotic lesions in vitro and in animal models, respectively. Humulus japonicas therefore, appears to be effective against the development of atherosclerosis, so it may have a potential therapeutic application in atherosclerosis treatment (9).

\section{Artemisia judaica $L$.}

Artemisia judaica L. is a medicinal herb which is specifically found in the southern desert of Jordan near to the Jordan-Saudi Arabia borders and in Wadi Araba in Southern Badia. A Study by Abu-Darwish et al., in 2016, revealed that safe concentrations of Artemisia judaica essential oil effectively suppress NO production evoked by lipopolysaccharide in macrophages, emphasizing the possible anti-inflammatory properties of Artemisia judaica (31).

\section{Salvia miltiorrhiza}

Salvia miltiorrhiza var. alba is a perennial plant in the genus Salvia. This herb is highly valued in traditional Chinese medicine. It has been used for many years in traditional Chinese medicine to treat cardiovascular diseases associated with inflammation including atherosclerosis. Ma et al., (2016) demonstrated that the root extract of Salvia miltiorrhiza effectively suppressed the expression of inflammatory factors including TNF- $\alpha$, IL- $1 \beta$, and IL-8 proposing a potential application of this herb in diseases like atherosclerosis due to its anti-inflammatory properties (32). 


\section{Prosopis farcta}

Syrian mesquite (Prosopis farcta) is a species of the genus Prosopis and native to Asia, ranging from India to Iran. In herbal medicine, Prosopis farcta root is a suggested treatment for cardiovascular diseases. Saidi et al., (2016) investigated the efficacy of an aqueous extract of Prosopis farcta root on the development of experimental atherosclerosis in rabbits. According to their findings, serum lipid parameters including total cholesterol, HDL, TG, LDL, and VLDL levels were substantially increased in hypercholesterolemic animals. However, according to histopathological results, atherosclerotic plaque size was mimimally influenced. This outcome may reflect a decline of chest pain or the advantageous impacts of this plant root extract on cardiovascular health (33).

\section{Persimmon (Diospyros kaki L.)}

For many years, Persimmon leaves have been used to treat disorders like ischemic stroke, internal hemorrhage and atherosclerosis in traditional Chinese medicine. Xie et al., in a review on traditional uses of persimmon leaves published in 2015, declared that it could potentially be used as a therapeutic agent in cardiovascular disease treatment and prevention including atherosclerosis owing to its immunomodulatory and anti-inflammatory properties. However they proposed more investigation on the safety to use it as a natural drug in medicinal practices (34).

\section{Andrographis paniculata}

As an annual plant in the Acanthaceae family, Andrographis paniculata is widely found in Southern and Southeastern Asia. For many years, its main bioactive component called Andrographolide (AND) has been used in herbal remedies. According to different studies AND exerts its antiatherogenic properties by reducing VCAM-1 and ICAM-1 as two cell 
adhesion molecules which play a key role in migration of vascular smooth muscle cells (SMCs) (35).

\section{Plectranthus zeylanicus Benth}

Plectranthus zeylanicus Benth is a perennial herb cultivated in Sri Lanka and South India. People native to these two countries use it as a remedy to treat inflammatory diseases such as asthma and atherosclerosis. Napagoda et al., (2014) in their study revealed that Plectranthus zeylanicus Benth inhibited 5-lipoxygenase (5-LO). 5-LO has a key role in initiating the biosynthesis of pro-inflammatory leukotrienes, thus emphasizing the anti-inflammatory impact of this remedy in managing inflammatory associated diseases including atherosclerosis (36).

\section{Prunella vulgaris}

As a perennial herbaceous plant, Prunella vulgaris is widely distributed throughout the world. This herb belongs to the prunella genus, and for many years it has been used in herbal medicine to treat inflammatory diseases. A study published in 2013 indicated that Prunella vulgaris can reduce the expression of adhesion molecules including intercellular adhesion molecule-1, vascular cell adhesion molecule-1, E-selectin and also the activation of N1F-kB. Molecular analysis revealed that Prunella vulgaris exerts its anti-inflammatory effects by inhibiting the p38 MAPK/ERK signaling pathway (37).

\section{Phyllostachys edulis (Bamboo)}

For centuries, Bamboo leaves have been used as a food or a natural remedy in Asian countries like Japan and Korea. Owing to its anti-inflammatory and anti-oxidant activity, Bamboo leaves may be beneficial in treating cardiovascular diseases including atherosclerosis. In vitro investigation of Bamboo leaf extract effects on human umbilical vein 
endothelial cells (HUVECs) has shown that it is able to inhibit monocyte adhesion and vascular cell adhesion molecule-1 expression, reduce oxygen species generation and inhibit interleukin-6 secretion from lipopolysaccharide-stimulated monocytes (38).

\section{Panax notoginseng saponins}

Panax notoginseng saponins, also known as Chinese ginseng in English, has been used for many years as a natural remedy for cardiovascular diseases in Asian countries including China, Korea and Japan. Studying Panax notoginseng saponins (PNS) effects on atherosclerosis in rats has indicated that PNS upregulates liver X receptor alpha (LXR $\alpha)$ expression and consequent ABCA1 and ABCG1 overexpression and it also inhibits $\mathrm{NF}-\mathrm{\kappa B}$ DNA binding activity. LXR $\alpha$ as a receptor, regulates macrophage function which is involved in lipid metabolism and inflammation (39).

PNS not only decreases lipids, serum Ox-LDL level as well as CD40 and MMP-9 expressions in apoE (-/-) mice (40), but also lessens the size of atherosclerotic plaques, enhances endothelialization (41) and suppresses the formation of foam cells (42).

Furthermore, a Ginsenoside derivative known as Ginsenoside Rd blocks voltage dependent $\mathrm{Ca}^{2}+$ channels and markedly decreases the size of atherosclerotic plaques and Ox-LDL in the apoE (-/-) rats. Moreover, the formation of foam cells is suppressed by Ginsenoside $\mathrm{Rd}$ in vitro (42). Conclusively, with regard to the anti-inflammatory effects of PNS and also its role in lipid metabolism regulation, this natural remedy might be introduced as a potential therapeutic agent in atherosclerosis treatment. 


\section{Astragalus mongholicus Bunge}

In traditional Chinese medicine, Astragalus mongholicus Bunge has been used for hundreds of years. Studies using animal models and clinical trials have demonstrated that this medicinal herb may be beneficial in atherosclerosis. Thus, an extract of Astragalus mongholicus Bunge lowered total cholesterol and triglyceride in the blood and also the levels of LDL cholesterol and apolipoprotein B. Moreover, Astragalus mongholicus Bunge extract reduced fatty streaks and the sclerosing index (43). Total flavonoids of Astragalus (TFA) as the major active ingredient in Astragalus mongholicus Bunge is thought to possess the main antioxidant activity of this extract in managing atherosclerosis (44).

\section{Perilla frutescens}

Perilla frutescens is a leafy vegetable cultivated widely in some Asian countries, including Japan, and China. This herb exists in two varieties: red and green. Exhibiting its antioxidant effect, Perilla frutescens reduces LDL oxidizability. As mentioned earlier, oxidative modification of LDL plays a crucial role in the pathogenesis of atherosclerosis and antioxidant activity can be induced by polyphenol compounds contained in the leaves of Perilla frutescens. Oral consumption of the red variety of Perilla frutescens results in a longer oxidation lag time and also a significant reduction in lipid peroxide formation as well as the electrophoretic mobility of LDL. Taken together, Perilla frutescens consumption can be beneficial for medicinal purposes against the development of atherosclerosis (45).

\section{Dachaihutang}

A study performed in 2009 by et al., in rabbits revealed that early treatment of arterial lesions led to a reduction of atherosclerosis size. Dachaihutang resulted in the regression of lesions and also prevented atherosclerosis development by upregulation of carnitine patmitoyl 
transferase-1(CPT-1) expression in vascular smooth muscle layers. The treated animal group also displayed a reduction in total cholesterol, triglyceride and LDL serum levels (46).

\section{Cortex moutan}

Cortex Moutan (CM, root bark of Paeonia suffruticosa Andrews) is widely used in traditional Chinese medicine due to its analgesic, antipyretic, and anti-inflammatory properties and also its ability to prevent thromboembolic diseases. Paeonol is one of the major active ingredients in Cortex Moutan which is thought to contribute to the anti-atherosclerotic effect of this remedy. Cortex Moutan exerts its anti-atherosclerotic activity by reducing atherosclerosis plaque formation and also by suppressing TNF- alpha, IL-1 beta, and CRP and the translocation of NF- kappaB to the nucleus (47).

\section{Ginkgo biloba}

Ginkgo biloba is largely cultivated and is native to China. To assess the effect of this popular traditional medicine, Pierre et al., (2008) conducted an in vitro study using an extract from Ginkgo biloba with well-known antioxidant properties. Ginkgo biloba extract Egb-761, a standardized formulation of Gingko leaf extract, protected adhesive properties so preventing endothelial dysfunction as a key event in atherosclerosis development. EGb-761 mediates an inhibition of increased lipoperoxide level by limiting the reduction of $\mathrm{Na}^{+} / \mathrm{K}^{+}$-ATPase activity induced by Ox-LDL in endothelial cells, thereby exerting its protective effect against atherosclerosis (48). 


\section{Lycopus lucidus}

Lycopus lucidus is widely used in oriental traditional medicine. Pretreatment of exposed- tohigh- glucose primary cultured human umbilical vein endothelial cells (HUVEC) with aqueous extract of the leaves of L. lucidus Turcz. (ALT) demonstrated that CAMs expression was significantly attenuated and the enhanced cell adhesion between monocyte and HUVEC was blocked. In addition, translocation and promoter transcriptional activity of NF-kappaB was suppressed. All of these consequences can contribute to prevention and treatment of atherosclerosis as a major complication of diabetes mellitus (49).

\section{Zingiber Officinale Rosc (ginger)}

Zingiber officinale Rosc is widely used as a spice and also in Chinese traditional medicine and other traditional medicinal systems as a herbal medication. Surveys have indicated that ginger in atherosclerosis exerts its effect by decreasing plasma lipid levels and the size of atherosclerotic lesions and these properties are due to antioxidative activity. This plant could have an inhibitory or scavenging effect on radicals by increasing GSH-Px and reducing LPO (50).

\section{Garlic}

Garlic has been widely used to prevent and treat cardiovascular diseases for many years. Sallyl cysteine (SAC) is the main constituent of garlic that inhibits NO production by suppression of iNOS mRNA and protein expression. Moreover, by suppressing the production of hydroxyl radicals, garlic extract demonstrates antioxidant activity. These properties make garlic an effective natural medication against inflammatory based diseases including atherosclerosis (51). 


\section{Quercus infectoria}

Quercus infectoria is native to southern Europe and the Middle East. This plant has high antioxidant activity by inhibiting Ox-LDL production thereby preventing atherosclerosis development.

Furthermore, Quercus infectoria extract reduces the level of total cholesterol, triglyceride and LDL in plasma, emphasizing its contribution in atherosclerosis prevention and treatment (52).

\section{Conclusion:}

Although, using synthetic compounds to treat and prevent diseases is attractive, these types of medications can cause side effects. According to preclinical and clinical studies, natural herbs represent potential therapeutic agents in the prevention and treatment of atherosclerosis. Atherosclerosis is the crosstalk of lipid metabolism and inflammation. Medicinal plants may have various properties extended from antioxidant and antihyperlipidemic properties to antiendothelial dysfunction activity. These features introduce them as potential medications against atherosclerosis. However, sometimes the exact underlying mechanisms have not been precisely determined or understood. These herbs bear promising therapeutic potential to develop safer and less expensive medications to prevent and treat various conditions including atherosclerosis. However, their safety needs to be explored in more detail.

\section{References}

1. Moss JW, Ramji DP. Cytokines: roles in atherosclerosis disease progression and potential therapeutic targets. Future Med Chem. 2016 Jul;8(11):1317-30.

2. WHO. World Health Organization Fact Sheet 317. 2015. [Internet]. Available from: http://www.who.int/mediacentre/factsheets/fs317/en/.

3. Moss JWE, Williams JO, Ramji DP. Nutraceuticals as therapeutic agents for atherosclerosis. Biochim Biophys Acta. 2018 May;1864(5 Pt A):1562-72. 
4. Benjamin EJ, Blaha MJ, Chiuve SE, Cushman M, Das SR, Deo R, et al. Heart Disease and Stroke Statistics-2017 Update: A Report From the American Heart Association. Circulation. 2017 Mar 7;135(10):e146-e603.

5. Buckley ML, Ramji DP. The influence of dysfunctional signaling and lipid homeostasis in mediating the inflammatory responses during atherosclerosis. Biochim Biophys Acta. 2015 Jul;1852(7):1498-510.

6. Zernecke A, Weber C. Chemokines in atherosclerosis: proceedings resumed. Arterioscler Thromb Vasc Biol. 2014 Apr;34(4):742-50.

7. Ross R. Atherosclerosis--an inflammatory disease. N Engl J Med. 1999 Jan 14;340(2):11526.

8. Braunwald E. Shattuck lecture--cardiovascular medicine at the turn of the millennium: triumphs, concerns, and opportunities. N Engl J Med. 1997 Nov 6;337(19):1360-9.

9. Lim H, Noh JR, Kim YH, Hwang JH, Kim KS, Choi DH, et al. Anti-atherogenic effect of Humulus japonicus in apolipoprotein E-deficient mice. Int J Mol Med. 2016 Oct;38(4):110110.

10. Pac-Kozuchowska E, Krawiec P, Grywalska E. Selected risk factors for atherosclerosis in children and their parents with positive family history of premature cardiovascular diseases: a prospective study. BMC Pediatr. 2018 Apr 3;18(1):123.

11. Berenson GS. Cardiovascular risk begins in childhood: a time for action. Am J Prev Med. 2009 Jul;37(1 Suppl):S1-2.

12. Weber C, Noels H. Atherosclerosis: current pathogenesis and therapeutic options. Nat Med. 2011 Nov 7;17(11):1410-22.

13. Libby $\mathrm{P}$, Lichtman $\mathrm{AH}$, Hansson GK. Immune effector mechanisms implicated in atherosclerosis: from mice to humans. Immunity. 2013 Jun 27;38(6):1092-104.

14. Yamashita T, Sasaki N, Kasahara K, Hirata K. Anti-inflammatory and immunemodulatory therapies for preventing atherosclerotic cardiovascular disease. J Cardiol. 2015 Jul;66(1):1-8.

15. Nicolaou G, Erridge C. Toll-like receptor-dependent lipid body formation in macrophage foam cell formation. Curr Opin Lipidol. 2010 Oct;21(5):427-33.

16. Libby P. Mechanisms of acute coronary syndromes and their implication for therapy. N Engl J Med 2013;368:2004-13.

17. Bentzon JF, Otsuka F, Virmani R, Falk E. Mechanisms of plaque formation and rupture. Circ Res 2014;114:1852-66.

18. Libby P. Inflammation in atherosclerosis. Arterioscler Thromb Vasc Biol. 2012 Sep;32(9):2045-51.

19. Fava C, Montagnana M. Atherosclerosis Is an Inflammatory Disease which Lacks a Common Anti-inflammatory Therapy: How Human Genetics Can Help to This Issue. A Narrative Review. Front Pharmacol. 2018;9:55.

20. Sultana S, Asif HM. Review: Medicinal plants combating against hypertension: A green antihypertensive approach. Pak J Pharm Sci. 2017 Nov;30(6):2311-9.

21. Back M, Hansson GK. Anti-inflammatory therapies for atherosclerosis. Nat Rev Cardiol. 2015 Apr;12(4):199-211.

22. Roubille F, Kritikou EA, Roubille C, Tardif JC. Emerging anti-inflammatory therapies for atherosclerosis. Curr Pharm Des. 2013;19(33):5840-9.

23. Sundar Dhilip Kumar S, Houreld NN, Abrahamse H. Therapeutic Potential and Recent Advances of Curcumin in the Treatment of Aging-Associated Diseases. Molecules. 2018 Apr $5 ; 23(4)$. 
24. Lee HS, Lee MJ, Kim H, Choi SK, Kim JE, Moon HI, et al. Curcumin inhibits TNFalphainduced lectin-like oxidised LDL receptor-1 (LOX-1) expression and suppresses the inflammatory response in human umbilical vein endothelial cells (HUVECs) by an antioxidant mechanism. J Enzyme Inhib Med Chem. 2010 Oct;25(5):720-9.

25. Liu W, Guo W, Guo L, Gu Y, Cai P, Xie N, et al. Andrographolide sulfonate ameliorates experimental colitis in mice by inhibiting Th1/Th17 response. Int Immunopharmacol. 2014 Jun;20(2):337-45.

26. Amano Y, Shimada M, Miura S, Adachi R, Tozawa R. Antidyslipidemic effects of a farnesoid X receptor antagonist in primates. Life Sci. 2014 Jun 13;106(1-2):25-31.

27. Leyes P, Martinez E, Larrousse M, Cofan M, Trabal J, Perez-Heras AM, et al. Effects of ezetimibe on cholesterol metabolism in HIV-infected patients with protease inhibitorassociated dyslipidemia: a single-arm intervention trial. BMC Infect Dis. 2014 Sep $11 ; 14: 497$.

28. Fidele N, Joseph B, Emmanuel T, Theophile D. Hypolipidemic, antioxidant and antiatherosclerogenic effect of aqueous extract leaves of Cassia. occidentalis Linn (Caesalpiniaceae) in diet-induced hypercholesterolemic rats. BMC Complement Altern Med. 2017 Jan 25;17(1):76.

29. Saraf S, Dixit VK, Tripathi SC, Patnaik GK. Antihepatotoxic Activity of Cassia occidentalis. Pharm Biol. 1994;32:178-83.

30. Hosseinpour-Jaghdani F, Shomali T, Gholipour-Shahraki S, Rahimi-Madiseh M, Rafieian-Kopaei M. Cornus mas: a review on traditional uses and pharmacological properties. J Complement Integr Med. 2017 Mar 31;14(3).

31. Abu-Darwish MS, Cabral C, Goncalves MJ, Cavaleiro C, Cruz MT, Zulfiqar A, et al. Chemical composition and biological activities of Artemisia judaica essential oil from southern desert of Jordan. J Ethnopharmacol. 2016 Sep 15;191:161-8.

32. Ma S, Zhang D, Lou H, Sun L, Ji J. Evaluation of the anti-inflammatory activities of tanshinones isolated from Salvia miltiorrhiza var. alba roots in THP-1 macrophages. J Ethnopharmacol. 2016 Jul 21;188:193-9.

33. Saidi MR, Farzaei MH, Miraghaee S, Babaei A, Mohammadi B, Bahrami MT, et al. Antihyperlipidemic Effect of Syrian Mesquite (Prosopis farcta) Root in High Cholesterol Diet-Fed Rabbits. J Evid Based Complementary Altern Med. 2016 Oct;21(4):NP62-6.

34. Xie C, Xie Z, Xu X, Yang D. Persimmon (Diospyros kaki L.) leaves: a review on traditional uses, phytochemistry and pharmacological properties. J Ethnopharmacol. 2015 Apr 2;163:229-40.

35. Al Batran R, Al-Bayaty F, Al-Obaidi MM, Ashrafi A. Insights into the antiatherogenic molecular mechanisms of andrographolide against Porphyromonas gingivalis-induced atherosclerosis in rabbits. Naunyn Schmiedebergs Arch Pharmacol. 2014 Dec;387(12):114152.

36. Napagoda M, Gerstmeier J, Wesely S, Popella S, Lorenz S, Scheubert K, et al. Inhibition of 5-lipoxygenase as anti-inflammatory mode of action of Plectranthus zeylanicus Benth and chemical characterization of ingredients by a mass spectrometric approach. $\mathbf{J}$ Ethnopharmacol. 2014 Feb 3;151(2):800-9.

37. Park SH, Koo HJ, Sung YY, Kim HK. The protective effect of Prunella vulgaris ethanol extract against vascular inflammation in TNF-alpha-stimulated human aortic smooth muscle cells. BMB Rep. 2013 Jul;46(7):352-7.

38. Choi S, Park MS, Lee YR, Lee YC, Kim TW, Do SG, et al. A standardized bamboo leaf extract inhibits monocyte adhesion to endothelial cells by modulating vascular cell adhesion protein-1. Nutr Res Pract. 2013 Feb;7(1):9-14. 
39. Christoffolete MA, Doleschall M, Egri P, Liposits Z, Zavacki AM, Bianco AC, et al. Regulation of thyroid hormone activation via the liver $\mathrm{X}$-receptor/retinoid $\mathrm{X}$-receptor pathway. J Endocrinol. 2010 May;205(2):179-86.

40. Liu G, Wang B, Zhang J, Jiang H, Liu F. Total panax notoginsenosides prevent atherosclerosis in apolipoprotein E-knockout mice: Role of downregulation of CD40 and MMP-9 expression. J Ethnopharmacol. 2009 Nov 12;126(2):350-4.

41. Liu Y, Hao F, Zhang H, Cao D, Lu X, Li X. Panax notoginseng saponins promote endothelial progenitor cell mobilization and attenuate atherosclerotic lesions in apolipoprotein E knockout mice. Cell Physiol Biochem. 2013;32(4):814-26.

42. Li J, Xie ZZ, Tang YB, Zhou JG, Guan YY. Ginsenoside-Rd, a purified component from panax notoginseng saponins, prevents atherosclerosis in apoE knockout mice. Eur $\mathbf{J}$ Pharmacol. 2011 Feb 10;652(1-3):104-10.

43. Lu Y, Li JZ, Zheng X. [Effect of Astragalus Angelica mixture on serum lipids and glomerulosclerosis in rats with nephrotic syndrome]. Zhongguo Zhong Xi Yi Jie He Za Zhi. 1997 Aug;17(8):478-80.

44. Wang D, Zhuang Y, Tian Y, Thomas GN, Ying M, Tomlinson B. Study of the effects of total flavonoids of Astragalus on atherosclerosis formation and potential mechanisms. Oxid Med Cell Longev. 2012;2012:282383.

45. Saita E, Kishimoto Y, Tani M, Iizuka M, Toyozaki M, Sugihara N, et al. Antioxidant activities of Perilla frutescens against low-density lipoprotein oxidation in vitro and in human subjects. J Oleo Sci. 2012;61(3):113-20.

46. Xuan L, Li X, Sheng X, Xu M, Che X. [Effect of dachaihutang on expression of carnitine patmitoryl transferase-1 in vascular smooth muscle layer of atherosclerotic rabbits]. Zhongguo Zhong Yao Za Zhi. 2009 Sep;34(17):2232-5.

47. Li H, Dai M, Jia W. Paeonol attenuates high-fat-diet-induced atherosclerosis in rabbits by anti-inflammatory activity. Planta Med. 2009 Jan;75(1):7-11.

48. Pierre SV, Lesnik P, Moreau M, Bonello L, Droy-Lefaix MT, Sennoune S, et al. The standardized Ginkgo biloba extract Egb-761 protects vascular endothelium exposed to oxidized low density lipoproteins. Cell Mol Biol (Noisy-le-grand). 2008 Oct 26;54 Suppl:OL1032-42.

49. Lee YJ, Kang DG, Kim JS, Lee HS. Lycopus lucidus inhibits high glucose-induced vascular inflammation in human umbilical vein endothelial cells. Vascul Pharmacol. 2008 Jan;48(1):38-46.

50. Liu N, Huo G, Zhang L, Zhang X. [Effect of Zingiber OfficinaleRosc on lipid peroxidation in hyperlipidemia rats]. Wei Sheng Yan Jiu. 2003 Jan;32(1):22-3.

51. Kim KM, Chun SB, Koo MS, Choi WJ, Kim TW, Kwon YG, et al. Differential regulation of NO availability from macrophages and endothelial cells by the garlic component S-allyl cysteine. Free Radic Biol Med. 2001 Apr 1;30(7):747-56.

52. Gholamhoseinian A, Shahouzehi B, Joukar S, Iranpoor M. Effect of Quercus infectoria and Rosa damascena on lipid profile and atherosclerotic plaque formation in rabbit model of hyperlipidemia. Pak J Biol Sci. 2012 Jan 1;15(1):27-33. 